\title{
Some Remarks on a Class of Finite Projective Klingenberg Planes
}

\author{
Atilla Akpinara İsa Doğan ${ }^{b}$, Elif Demirci ${ }^{b}$, Zeynep Sena Gürel ${ }^{b}$, Bercem Boztemurb, \\ aUniversity of Uludağ, Faculty of Science and Art, Department of Mathematics, 16059, Bursa, Turkey \\ bUniversity of Uludağ, Institute of Science, Department of Mathematics, 16059, Bursa, Turkey
}

\begin{abstract}
In this article, we deal with a class of projective Klingenberg planes constructed over a plural algebra of order $\mathrm{m}$. Thanks to this, the incidence matrices for some special cases of the class are obtained. Next, the number of collineations of the certain classes are found. Besides, an example of a collineation for these classes are given. Finally, we achieve to carry the obtained results to more general case.
\end{abstract}

Indexing terms/Keywords: Plural Algebra, Local Ring, Projective Klingenberg Plane, Projective Collineation

Subject Classification: 51C05 [Ring geometry], 51N35 [Questions of classical algebraic geometry], 51A45 [Incidence structures imbeddable into projective geometries]

Type (Method/Approach): We take the finite field $\mathbb{Z}_{p}$ and the finite local ring $\mathbb{Z}_{p^{r}}$ instead of the field of real number in the definition of real plural algebra of order $\mathrm{m}$. Such an obtained algebra has the structure of a local ring. We know that a plane coordinatized by the local ring is a projective Klingenberg plane. So, we obtain some numerical results by studying the incidence matrices and collineations on classes of the planes.

Date of Publication: 2018-08-30

DOI: https://doi.org/10.24297/jam.v14i2.7527

ISSN: 2347-1921

Volume: 14 Issue: 02

Journal: Journal of Advances in Mathematics

Publisher: CIRWORLD

Website: https://cirworld.com

This work is licensed under a Creative Commons Attribution 4.0 International License. 


\section{Introduction}

Jukl, in [1], study on the real plural algebra of order $m$ and so investigated the linear forms on a free finite dimensional module $\mathrm{M}$, especially their kernel. Jukl continued to deal with free finite dimensional modules in [2]. In [3], Erdogan et. al. examined some properties of the modules constructed over the real plural algebra and later, in [4], Ciftci and Erdogan established an n-dimensional projective coordinate space over $(n+1)$ dimensional module constructed by the help of this real plural algebra. For more detailed information on modules, see [5]. For the algebraic notions that will be used throughout this article, we refer to [6] and [7].

In this article we will study on a class of projective Klingenberg (PK) plane $\mathrm{M}(\mathbf{A})$ constructed over the algebra $\mathbf{A}:=F+F \eta+F \eta^{2}+\cdots+F \eta^{m-1}$ such that $\eta^{m}=0$ for $\eta \notin F$ (where $F$ is a field). So, the incidence matrices for some special cases of the class are obtained, by taking the field $\mathbb{Z}_{p}$ (where $p$ is a prime) instead of $F$. Also, the number of collineations of these classes are found. Besides, an example of a collineation for the classes are given. Finally, the obtained results are carried over PK plane $\mathrm{M}(\boldsymbol{A})$, which is more general case than $\mathrm{M}(\mathbf{A})$, constructed over the algebra $\mathcal{A}$ of order $\mathrm{m}$ obtained by taking the local ring $\mathbb{Z}_{\mathrm{p}}$ (where $\mathrm{r}$ is a positive integer) instead of $\mathrm{F}$.

\section{Preliminaries}

In this section we will give some definitions and results from [1], [8] and [9], which will be the basis of this study.

Definition 1 ([1, Def. 1]) The real plural algebra of order $m$ is every linear algebra $A$ on $\mathbb{R}$ having as a vector space over $\mathbb{R}$ a basis $\left\{1, \eta, \eta^{2}, \cdots, \eta^{\mathrm{m}-1}\right\}$ where $\eta^{\mathrm{m}}=0$ for $\eta \notin \mathbb{R}$.

By Definition 1, we see that an element $x$ of $A$ is of the form $x=a_{0}+a_{1} \eta+a_{2} \eta^{2}+\cdots+a_{m-1} \eta^{m-1}$ where $a_{i} \in \mathbb{R}$ for $0 \leq i \leq m-1$.

Now we can state the following two results without proof.

Proposition 2 ([1, Prop 1.3]) An element $x=a_{0}+a_{1} \eta+a_{2} \eta^{2}+\cdots+a_{m-1} \eta^{m-1} \in A$ is a unit if and only if $a_{0} \neq 0$.

Proposition 3 ([1, Prop 1.5]) $A$ is a local ring with the maximal ideal $\eta A$. The subsets $\eta^{j} A, 1 \leq j \leq m$, are all ideals in $A$. In $\left[1\right.$, Prop 1.7], it is stated that $A$ is isomorphic to the linear algebra of matrix $M_{m m}(\mathbb{R})$ of the form

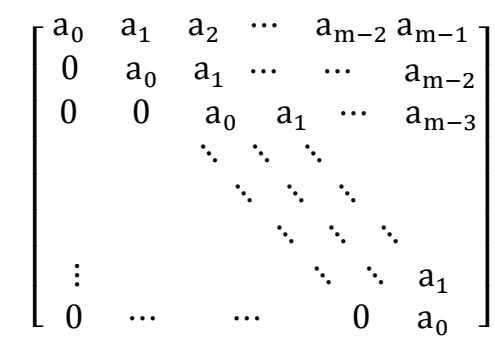

where $b_{i} \in \mathbb{R}$ for $0 \leq i \leq m-1$ (for the detailed proof of this fact, see [3].

Now, we will recall some information from [8].

Definition 4 Let $M=(\mathbf{P}, \mathbf{L}, \in, \sim)$ consist of an incidence structure $(\mathbf{P}, \mathbf{L}, \in)$ (points, lines, incidence) and an equivalence relation ' $\sim$ ' (neighbour relation) on $\mathbf{P}$ and on $\mathbf{L}$. Then $\mathbf{M}$ is called a projective Klingenberg plane (PK-plane), if it satisfies the following axioms:

(PK1) If $\mathrm{Q}$ and $\mathrm{S}$ are non-neighbour points, then there is a unique line $\mathrm{QS}$ through $\mathrm{Q}$ and $\mathrm{S}$. 
(PK2) If $\mathrm{g}$ and $\mathrm{h}$ are non-neighbour lines, then there is a unique point $\mathrm{g} \wedge \mathrm{h}$ on both $\mathrm{g}$ and $\mathrm{h}$.

(PK3) There is a projective plane $M^{*}=\left(\mathbf{P}^{*}, \mathbf{L}^{*}, \in\right)$ and incidence structure epimorphism $\Psi: M \rightarrow M^{*}$, such that the conditions

$$
\Psi(\mathrm{Q})=\Psi(\mathrm{S}) \Leftrightarrow \mathrm{Q} \sim \mathrm{S}, \Psi(\mathrm{g})=\Psi(\mathrm{h}) \Leftrightarrow \mathrm{g} \sim \mathrm{h}
$$

hold for all $Q, S \in \mathbf{P}, g, h \in \mathbf{L}$.

An incidence structure automorphism preserving and reflecting the neighbour relation is called a collineation of $\mathrm{M}$.

A ring $R$ with identity element 1 is called local if the set I of its non-unit elements is an ideal.

Let $\mathcal{R}$ be a local ring with the maximal ideal $\mathbf{I}$. Then $\mathbf{M}(\mathcal{R})=(\mathbf{P}, \mathbf{L}, \in, \sim)$ is the incidence structure with neighbour relation defined as follows:

$$
\begin{gathered}
\mathbf{P}=\{(\mathrm{x}, \mathrm{y}, 1): \mathrm{x}, \mathrm{y} \in \mathcal{R}\} \cup\{(1, \mathrm{y}, \mathrm{z}): \mathrm{y} \in \mathcal{R}, \mathrm{y} \in \mathrm{I}\} \cup\{(\mathrm{w}, 1, \mathrm{z}): \mathrm{w}, \mathrm{z} \in \mathbf{I}\} \\
\mathbf{L}=\{[\mathrm{m}, 1, \mathrm{k}]: \mathrm{m}, \mathrm{k} \in \mathcal{R}\} \cup\{[1, \mathrm{n}, \mathrm{t}]: \mathrm{t} \in \mathcal{R}, \mathrm{n} \in \mathbf{I}\} \cup\{[\mathrm{q}, \mathrm{n}, 1]: \mathrm{q}, \mathrm{n} \in \mathbf{I}\} \\
{[\mathrm{m}, 1, \mathrm{k}]=\{(\mathrm{x}, \mathrm{xm}+\mathrm{k}, 1): \mathrm{x} \in \mathcal{R}\} \cup\{(1, \mathrm{zk}+\mathrm{m}, \mathrm{z}): \mathrm{z} \in \mathbf{I}\}} \\
{[1, \mathrm{n}, \mathrm{t}]=\{(\mathrm{yn}+\mathrm{t}, \mathrm{y}, 1): \mathrm{y} \in \mathcal{R}\} \cup\{(\mathrm{zt}+\mathrm{n}, 1, \mathrm{z}): \mathrm{z} \in \mathbf{I}\}} \\
{[\mathrm{q}, \mathrm{n}, 1]\{(1, \mathrm{y}, \mathrm{yn}+\mathrm{q}): \mathrm{y} \in \mathcal{R}\} \cup\{(\mathrm{w}, 1, \mathrm{wq}+\mathrm{n}): \mathrm{w} \in \mathbf{I}\}} \\
\mathrm{S}=\left(\mathrm{x}_{1}, \mathrm{x}_{2}, \mathrm{x}_{3}\right) \sim\left(\mathrm{y}_{1}, \mathrm{y}_{2}, \mathrm{y}_{3}\right)=\mathrm{Q} \Leftrightarrow \mathrm{x}_{\mathrm{i}}-\mathrm{y}_{\mathrm{i}} \in \mathbf{I}(\mathrm{i}=1,2,3) \text { for } \forall \mathrm{S}, \mathrm{Q} \in \mathbf{P} . \\
\mathrm{g}=\left[\mathrm{x}_{1}, \mathrm{x}_{2}, \mathrm{x}_{3}\right] \sim\left[\mathrm{y}_{1}, \mathrm{y}_{2}, \mathrm{y}_{3}\right]=\mathrm{h} \Leftrightarrow \mathrm{x}_{\mathrm{i}}-\mathrm{y}_{\mathrm{i}} \in \mathbf{I}(\mathrm{i}=1,2,3) \text { for } \forall \mathrm{g}, \mathrm{h} \in \mathbf{L} .
\end{gathered}
$$

Now it is time to give the following theorem from [8].

Theorem $5 \mathrm{M}(\mathcal{R})$ is a PK-plane, and each desarguesian PK-plane is isomorphic to some $\mathrm{M}(\mathcal{R})$.

Now, from [9] we give the definition of an $n$-gon, which is meaningful when $n \geq 3$ : An $n$-tuple of pairwise nonneighbour points is called an (ordered) $n$-gon if no three of its elements are on neighbour lines.

\section{The Main Results}

Let $F$ be a field. Let $\eta^{m}=0$ for $\eta \notin F$. Consider $A:=F(\eta)=F+F \eta+F \eta^{2}+\cdots+\eta^{m-1}$ with componentwise addition and multiplication as follows:

$$
\begin{aligned}
a b= & \left(a_{0}+a_{1} \eta+a_{2} \eta^{2}+\ldots+a_{m-1} \eta^{m-1}\right)\left(b_{0}+b_{1} \eta+b_{2} \eta^{2}+\ldots+b_{m-1} n^{m-1}\right) \\
& =a_{0} b_{0}+\left(a_{1} b_{0}+b_{1} a_{0}\right) \eta+\left(a_{2} b_{0}+a_{1} b_{1}+a_{0} b_{2}\right) \eta^{2}+\ldots+\left(a_{m-1} b_{0}+a_{0} b_{m-1}\right) \eta^{m-1} \\
& =\sum_{k=0}^{m-1} \sum_{i+j=k}\left(a_{i} b_{j}\right) \eta^{k} \quad\left(a_{i}, b_{j} \in F \text { and } i, j=0,1,2,3, \ldots, m-1\right) .
\end{aligned}
$$

Then $\mathbf{A}$ is a (unital, commutative and associative) local ring with the maximal ideal $\mathbf{I}=\mathbf{A} n$ of non-units. So, by Theorem 5 we have that $\mathbf{M}(\mathbf{A})$ is a PK-plane. Also, by Definition 1, A can be called as plural F-algebra of order $\mathrm{m}$. 
Now we will obtain some results on this finite class of PK-plane by taking $\mathbb{Z}_{p}$ instead of $F$. If it is taken $\mathbb{Z}_{p}$ instead of $F$, then $|\mathbf{A}|=p^{m}$ and $|\mathbf{I}|=p^{m-1}$. The number of non-neighbour points and lines in $M(\mathbf{A})$ is $p^{2}+p+1$ and the number of points (or lines) in the neighborhood of any point (or any line) is $\left(\mathrm{p}^{\mathrm{m}-1}\right)^{2}$, respectively. Thus, the total number of points and lines in $\mathbf{M}(\mathbf{A})$ is $\left(p^{m-1}\right)^{2}\left(p^{2}+p+1\right)$. From this results, we see that the plane $\mathbf{M}$ stated in (PK3) of Definition 4 is a projective plane of order $p$.

For example, for $p=2$ and $m=2$, we can give the following results about the $P K-$ plane $M\left(\mathbb{Z}_{2}+\mathbb{Z}_{2} \eta\right)=(P, L, \in, \sim)$ :

The neighbour classes for points are

$N_{1}=(0,0,1) \sim(0, \eta, 1) \sim(\eta, 0,1) \sim(\eta, \eta, 1)$,

$\mathrm{N}_{2}=(0,1,1) \sim(\eta, 1,1) \sim(0,1+\eta, 1) \sim(\eta, 1+\eta, 1)$,

$N_{3}=(1,0,1) \sim(1, \eta, 1) \sim(1+\eta, 0,1) \sim(1+\eta, \eta, 1)$,

$\mathrm{N}_{4}=(1,1,1) \sim(1,1+\eta, 1) \sim(1+\eta, 1,1) \sim(1+\eta, 1+\eta, 1)$,

$N_{5}=(1,0,0) \sim(1,0, \eta) \sim(1, \eta, 0) \sim(1, \eta, \eta)$,

$\mathrm{N}_{6}=(1,1,0) \sim(1,1, \eta) \sim(1,1+\eta, 0) \sim(1,1+\eta, \eta)$,

$N_{7}=(0,1,0) \sim(0,1, \eta) \sim(\eta, 1,0) \sim(\eta, 1, \eta)$ where $|\mathbf{P}|=28$.

Similarly the neighbour classes for lines are

$d_{1}=[0,1,0] \sim[0,1, \eta] \sim[\eta, 1,0] \sim[\eta, 1, \eta]$,

$d_{2}=[0,1,1] \sim[0,1,1+\eta] \sim[\eta, 1,1] \sim[\eta, 1,1+\eta]$,

$d_{3}=[1,1,0] \sim[1,1, \eta] \sim[1+\eta, 1,0] \sim[1+\eta, 1, \eta]$

$\mathrm{d}_{4}=[1,1,1] \sim[1,1,1+\eta] \sim(1+\eta, 1,1) \sim(1+\eta, 1,1+\eta)$,

$d_{5}=[1,0,0] \sim[1,0, \eta] \sim[1, \eta, \eta] \sim[1, \eta, 0]$,

$d_{6}=[1,0,1] \sim[1,0,1+\eta] \sim[1, \eta, 1] \sim[1, \eta, 1+\eta]$,

$d_{7}=[0,0,1] \sim[0, \eta, 1] \sim[\eta, 0,1] \sim[\eta, \eta, 1]$ where $|\mathbf{L}|=28$.

The incidence relation $\in$ with the above results is given by the following incidence matrix (see Table 1): 


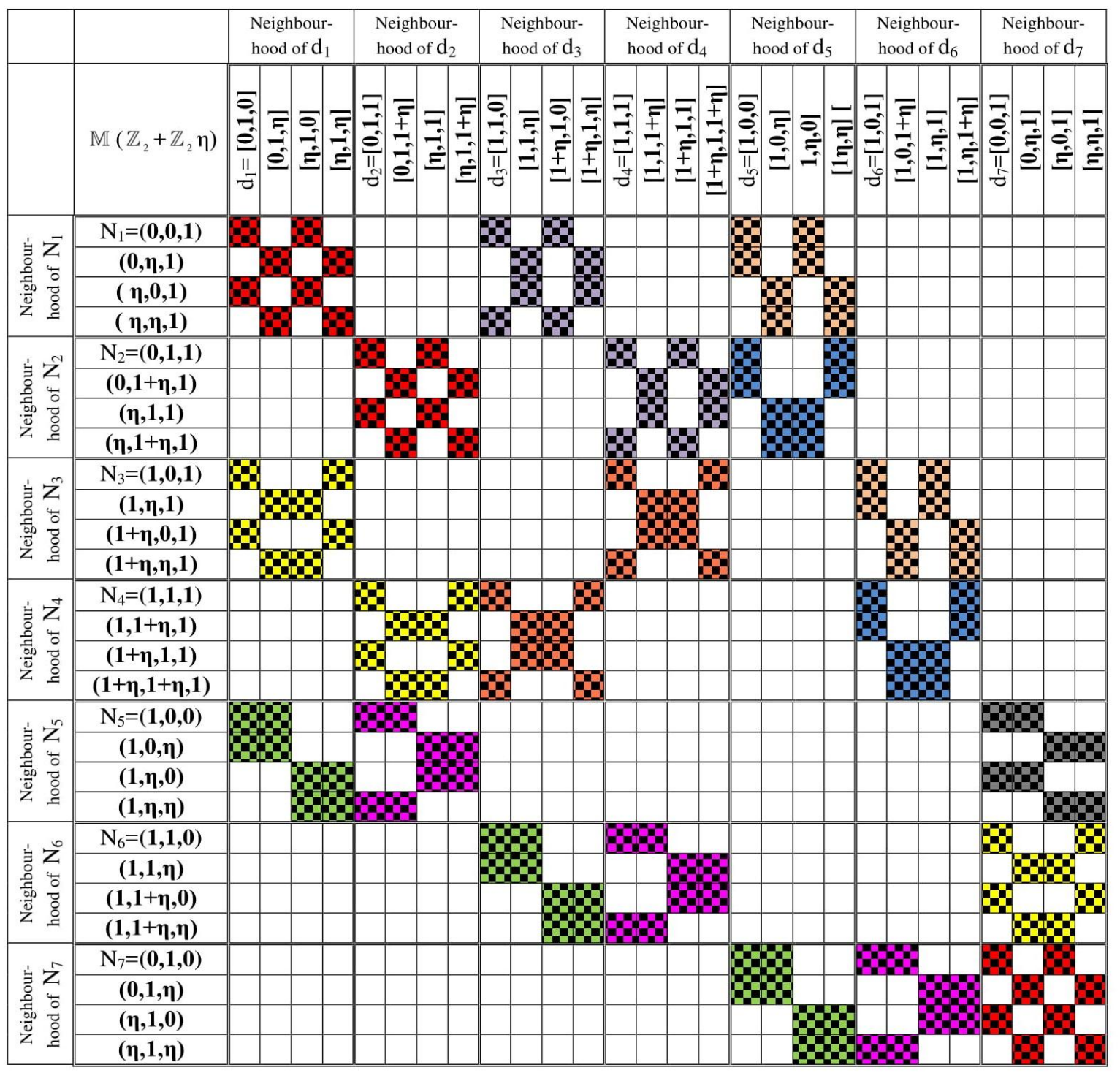

Table 1. The incidence matrix for the plane $M\left(\mathbb{Z}_{2}+\mathbb{Z}_{2} \eta\right)$

As a second example, for $p=2$ and $m=3$, we can give the following results about the PK-plane $M\left(\mathbb{Z}_{2}+\mathbb{Z}_{2} \eta+\mathbb{Z}_{2} \eta^{2}\right)=(\mathbf{P}, \mathbf{L}, \in, \sim)$ :

The neighbour classes for points are

$N_{1}=(0,0,1) \sim(0, \eta, 1) \sim\left(0, \eta^{2}, 1\right) \sim\left(0, \eta+\eta^{2}, 1\right) \sim(\eta, 0,1) \sim(\eta, \eta, 1) \sim\left(\eta, \eta^{2}, 1\right) \sim\left(\eta, \eta+\eta^{2}, 1\right) \sim\left(\eta^{2}, 0,1\right) \sim\left(\eta^{2}, \eta, 1\right) \sim$

$\left(\eta^{2}, \eta^{2}, 1\right) \sim\left(\eta^{2}, \eta+\eta^{2}, 1\right) \sim\left(\eta+\eta^{2}, 0,1\right) \sim\left(\eta+\eta^{2}, \eta, 1\right) \sim\left(\eta+\eta^{2}, \eta^{2}, 1\right) \sim\left(\eta+\eta^{2}, \eta+\eta^{2}, 1\right)$,

$N_{2}=(0,1,1) \sim(\eta, 1,1) \sim\left(\eta^{2}, 1,1\right) \sim\left(\eta+\eta^{2}, 1,1\right) \sim(0,1+\eta, 1) \sim(\eta, 1+\eta, 1) \sim\left(\eta^{2}, 1+\eta, 1\right) \sim\left(\eta+\eta^{2}, 1+\eta, 1\right) \sim\left(0,1+\eta^{2}, 1\right) \sim$

$\left(\eta, 1+\eta^{2}, 1\right) \sim\left(\eta^{2}, 1+\eta^{2}, 1\right) \sim\left(\eta+\eta^{2}, 1+\eta^{2}, 1\right) \sim\left(0,1+\eta+\eta^{2}, 1\right) \sim\left(\eta, 1+\eta+\eta^{2}, 1\right) \sim\left(\eta^{2}, 1+\eta+\eta^{2}, 1\right) \sim\left(\eta+\eta^{2}, 1+\eta+\eta^{2}, 1\right)$,

$N_{3}=(1,0,1) \sim(1, \eta, 1) \sim\left(1, \eta^{2}, 1\right) \sim\left(1, \eta+\eta^{2}, 1\right) \sim(1+\eta, 0,1) \sim(1+\eta, \eta, 1) \sim\left(1+\eta, \eta^{2}, 1\right) \sim\left(1+\eta, \eta+\eta^{2}, 1\right) \sim\left(1+\eta^{2}, 0,1\right) \sim$

$\left(1+\eta^{2}, \eta, 1\right) \sim\left(1+\eta^{2}, \eta^{2}, 1\right) \sim\left(1+\eta^{2}, \eta+\eta^{2}, 1\right) \sim\left(1+\eta+\eta^{2}, 0,1\right) \sim\left(1+\eta+\eta^{2}, \eta, 1\right) \sim\left(1+\eta+\eta^{2}, \eta^{2}, 1\right) \sim\left(1+\eta+\eta^{2}, \eta+\eta^{2}, 1\right)$,

$\mathrm{N}_{4}=(1,1,1) \sim(1,1+\eta, 1) \sim\left(1,1+\eta^{2}, 1\right) \sim\left(1,1+\eta+\eta^{2}, 1\right) \sim(1+\eta, 1,1) \sim(1+\eta, 1+\eta, 1) \sim\left(1+\eta, 1+\eta^{2}, 1\right) \sim$ 


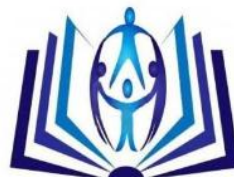

$\left(1+\eta, 1+\eta+\eta^{2}, 1\right) \sim\left(1+\eta^{2}, 1,1\right) \sim\left(1+\eta^{2}, 1+\eta, 1\right) \sim\left(1+\eta^{2}, 1+\eta^{2}, 1\right) \sim\left(1+\eta^{2}, 1+\eta+\eta^{2}, 1\right) \sim\left(1+\eta+\eta^{2}, 1,1\right) \sim$

$\left(1+\eta+\eta^{2}, 1+\eta, 1\right) \sim\left(1+\eta+\eta^{2}, 1+\eta^{2}, 1\right) \sim\left(1+\eta+\eta^{2}, 1+\eta+\eta^{2}, 1\right)$,

$N_{5}=(1,0,0) \sim(1,0, \eta) \sim\left(1,0, \eta^{2}\right) \sim\left(1,0, \eta+\eta^{2}\right) \sim(1, \eta, 0) \sim(1, \eta, \eta) \sim\left(1, \eta, \eta^{2}\right) \sim\left(1, \eta, \eta+\eta^{2}\right) \sim\left(1, \eta^{2}, 0\right) \sim\left(1, \eta^{2}, \eta\right) \sim$

$\left(1, \eta^{2}, \eta^{2}\right) \sim\left(1, \eta^{2}, \eta+\eta^{2}\right) \sim\left(1, \eta+\eta^{2}, 0\right) \sim\left(1, \eta+\eta^{2}, \eta\right) \sim\left(1, \eta+\eta^{2}, \eta^{2}\right) \sim\left(1, \eta+\eta^{2}, \eta+\eta^{2}\right)$,

$\mathrm{N}_{6}=(1,1,0) \sim(1,1, \eta) \sim\left(1,1, \eta^{2}\right) \sim\left(1,1, \eta+\eta^{2}\right) \sim(1,1+\eta, 0) \sim(1,1+\eta, \eta) \sim\left(1,1+\eta, \eta^{2}\right) \sim\left(1,1+\eta, \eta+\eta^{2}\right) \sim\left(1,1+\eta^{2}, 0\right) \sim$

$\left(1,1+\eta^{2}, \eta\right) \sim\left(1,1+\eta^{2}, \eta^{2}\right) \sim\left(1,1+\eta^{2}, \eta+\eta^{2}\right) \sim\left(1,1+\eta+\eta^{2}, 0\right) \sim\left(1,1+\eta+\eta^{2}, \eta\right) \sim\left(1,1+\eta+\eta^{2}, \eta^{2}\right) \sim$

$\left(1,1+\eta+\eta^{2}, \eta+\eta^{2}\right)$,

$N_{7}=(0,1,0) \sim(0,1, \eta) \sim\left(0,1, \eta^{2}\right) \sim\left(0,1, \eta+\eta^{2}\right) \sim(\eta, 1,0) \sim(\eta, 1, \eta) \sim\left(\eta, 1, \eta^{2}\right) \sim\left(\eta, 1, \eta+\eta^{2}\right) \sim\left(\eta^{2}, 1,0\right) \sim\left(\eta^{2}, 1, \eta\right) \sim$

$\left(\eta^{2}, 1, \eta^{2}\right) \sim\left(\eta^{2}, 1, \eta+\eta^{2}\right) \sim\left(\eta+\eta^{2}, 1,0\right) \sim\left(\eta+\eta^{2}, 1, \eta\right) \sim\left(\eta+\eta^{2}, 1, \eta^{2}\right) \sim\left(\eta+\eta^{2}, 1, \eta+\eta^{2}\right)$ where $|\mathbf{P}|=112$.

The neighbour classes for lines (where $|\mathbf{L}|=112$ ) can be similarly written. The incidence relation $\in$ of the line $[0,1,0]$ with the above results is given by the following incidence matrix (see Table 2):
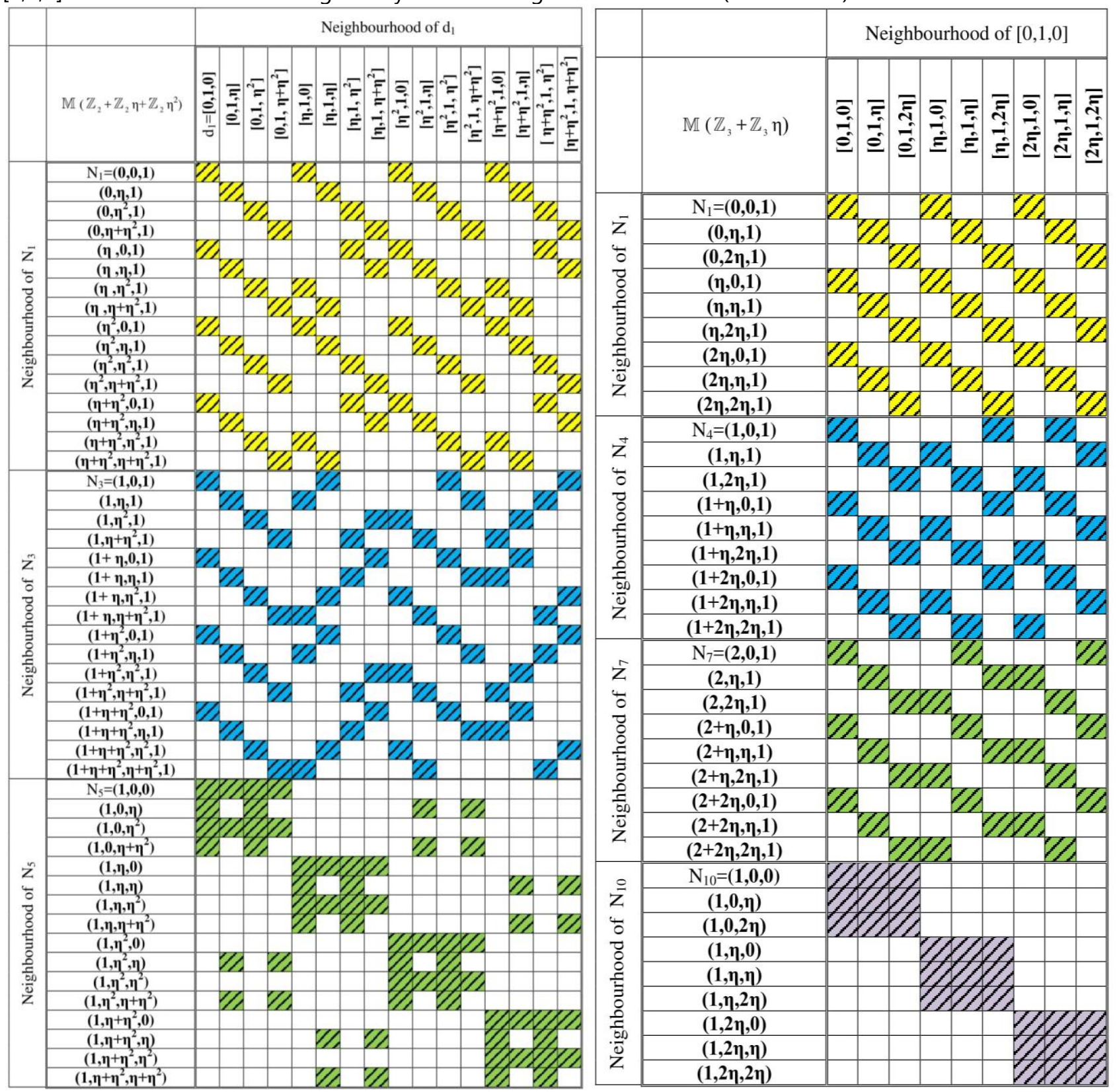

Table 2. The incidence matrix for the

Table 3. The incidence matrix for the line $[0,1,0]$ 
As a last example, for $p=3$ and $m=2$, we can give the following results about the PK-plane $\mathrm{M}\left(\mathbb{Z}_{3}+\mathbb{Z}_{3} \eta\right)=(\mathbf{P}, \mathbf{L}, \in, \sim)$ :

The neighbour classes for points are

$$
\begin{aligned}
N_{1}= & (0 \sim 0 \sim 1) \sim(0, \eta, 1) \sim(0,2 \eta, 1) \sim(\eta, 0,1) \sim(\eta, \eta, 1) \sim(\eta, 2 \eta, 1) \sim(2 \eta, 0,1) \sim(2 \eta, \eta, 1) \sim(2 \eta, 2 \eta, 1), \\
N_{2}= & (0,1,1) \sim(0,1+\eta, 1) \sim(0,1+2 \eta, 1) \sim(\eta, 1,1) \sim(\eta, 1+\eta, 1) \sim(\eta, 1+2 \eta, 1) \sim(2 \eta, 1,1) \sim(2 \eta, 1+\eta, 1) \sim(2 \eta, 1+2 \eta, 1), \\
N_{3}= & (0,2,1) \sim(0,2+\eta, 1) \sim(0,2+2 \eta, 1) \sim(\eta, 2,1) \sim(\eta, 2+\eta, 1) \sim(\eta, 2+2 \eta, 1) \sim(2 \eta, 2,1) \sim(2 \eta, 2+\eta, 1) \sim(2 \eta, 2+2 \eta, 1), \\
N_{4}= & (1,0,1) \sim(1, \eta, 1) \sim(1,2 \eta, 1) \sim(1+\eta, 0,1) \sim(1+\eta, \eta, 1) \sim(1+\eta, 2 \eta, 1) \sim(1+2 \eta, 0,1) \sim(1+2 \eta, \eta, 1) \sim(1+2 \eta, 2 \eta, 1), \\
N_{5}= & (1,1,1) \sim(1,1+\eta, 1) \sim(1,1+2 \eta, 1) \sim(1+\eta, 1,1) \sim(1+\eta, 1+\eta, 1) \sim(1+\eta, 1+2 \eta, 1) \sim(1+2 \eta, 1,1) \sim \\
& (1+2 \eta, 1+\eta, 1) \sim(1+2 \eta, 1+2 \eta, 1), \\
N_{6}= & (1,2,1) \sim(1,2+\eta, 1) \sim(1,2+2 \eta, 1) \sim(1+\eta, 2,1) \sim(1+\eta, 2+\eta, 1) \sim(1+\eta, 2+2 \eta, 1) \sim(1+2 \eta, 2,1) \sim \\
& (1+2 \eta, 2+\eta, 1) \sim(1+2 \eta, 2+2 \eta, 1), \\
N_{7}= & (2,0,1) \sim(2, \eta, 1) \sim(2,2 \eta, 1) \sim(2+\eta, 0,1) \sim(2+\eta, \eta, 1) \sim(2+\eta, 2 \eta, 1) \sim(2+2 \eta, 0,1) \sim(2+2 \eta, \eta, 1) \sim(2+2 \eta, 2 \eta, 1), \\
N_{8}= & (2,1,1) \sim(2,1+\eta, 1) \sim(2,1+2 \eta, 1) \sim(2+\eta, 1,1) \sim(2+\eta, 1+\eta, 1) \sim(2+\eta, 1+2 \eta, 1) \sim(2+2 \eta, 1,1) \sim \\
& (2+2 \eta, 1+\eta, 1) \sim(2+2 \eta, 1+2 \eta, 1), \\
N_{9}= & (2,2,1) \sim(2,2+\eta, 1) \sim(2,2+2 \eta, 1) \sim(2+\eta, 2,1) \sim(2+\eta, 2+\eta, 1) \sim(2+\eta, 2+2 \eta, 1) \sim(2+2 \eta, 2,1) \sim \\
& (2+2 \eta, 2+\eta, 1) \sim(2+2 \eta, 2+2 \eta, 1), \\
N_{10}= & (1,0,0) \sim(1,0, \eta) \sim(1,0,2 \eta) \sim(1, \eta, 0) \sim(1, \eta, \eta) \sim(1, \eta, 2 \eta) \sim(1,2 \eta, 0) \sim(1,2 \eta, \eta) \sim(1,2 \eta, 2 \eta), \\
N_{11}= & (1,1,0) \sim(1,1, \eta) \sim(1,1,2 \eta) \sim(1,1+\eta, 0) \sim(1,1+\eta, \eta) \sim(1,1+\eta, 2 \eta) \sim(1,1+2 \eta, 0) \sim(1,1+2 \eta, \eta) \sim(1,1+2 \eta, 2 \eta), \\
N_{12}= & (1,2,0) \sim(1,2, \eta) \sim(1,2,2 \eta) \sim(1,2+\eta, 0) \sim(1,2+\eta, \eta) \sim(1,2+\eta, 2 \eta) \sim(1,2+2 \eta, 0) \sim(1,2+2 \eta, \eta) \sim(1,2+2 \eta, 2 \eta) \\
N_{13}= & (0,1,0) \sim(0,1, \eta) \sim(0,1,2 \eta) \sim(\eta, 1,0) \sim(\eta, 1, \eta) \sim(\eta, 1,2 \eta) \sim(2 \eta, 1,0) \sim(2 \eta, 1, \eta) \sim(2 \eta, 1,2 \eta) w h e r e|P|=117 .
\end{aligned}
$$

The neighbour classes for lines (where $|\mathbf{L}|=117$ ) can be easily obtained as in the first example. The incidence relation $\in$ of the line $[0,1,0]$ with the above results is given by the following incidence matrix (see Table 3 ).

Notice that the plane $M{ }^{*}$ in (PK3) of Definition 4 for the plane $M\left(\mathbb{Z}_{2}+\mathbb{Z}_{2} \eta\right)$ is the Fano plane of order 2, the smallest finite projective plane. The obtained plane $M\left(\mathbb{Z}_{2}+\mathbb{Z}_{2} \eta\right)$ is not isomorphic to the finite projective Klingenberg plane $M\left(\mathbb{Z}_{4}\right)$ (see Table 4 for the incidence table) coordinatized by $\mathbb{Z}_{4}=\{0,1,2,3\}$ with the maximal ideal $I=\{0,2\}$ in [10]. For this, it is enough to check the incidence relations $N_{3} \in d_{4}$ in Table 1 and Table 4 since the other incidence relations are the same. The fact that the planes $M\left(\mathbb{Z}_{2}+\mathbb{Z}_{2} \eta\right)$ and $M\left(\mathbb{Z}_{4}\right)$ are not isomorphic is not new and can be found in the literature at different places, see e.g. [11,12]. Moreover, it can be seen to the papers of $[13,14,15]$ for more detailed information about such finite structures. 


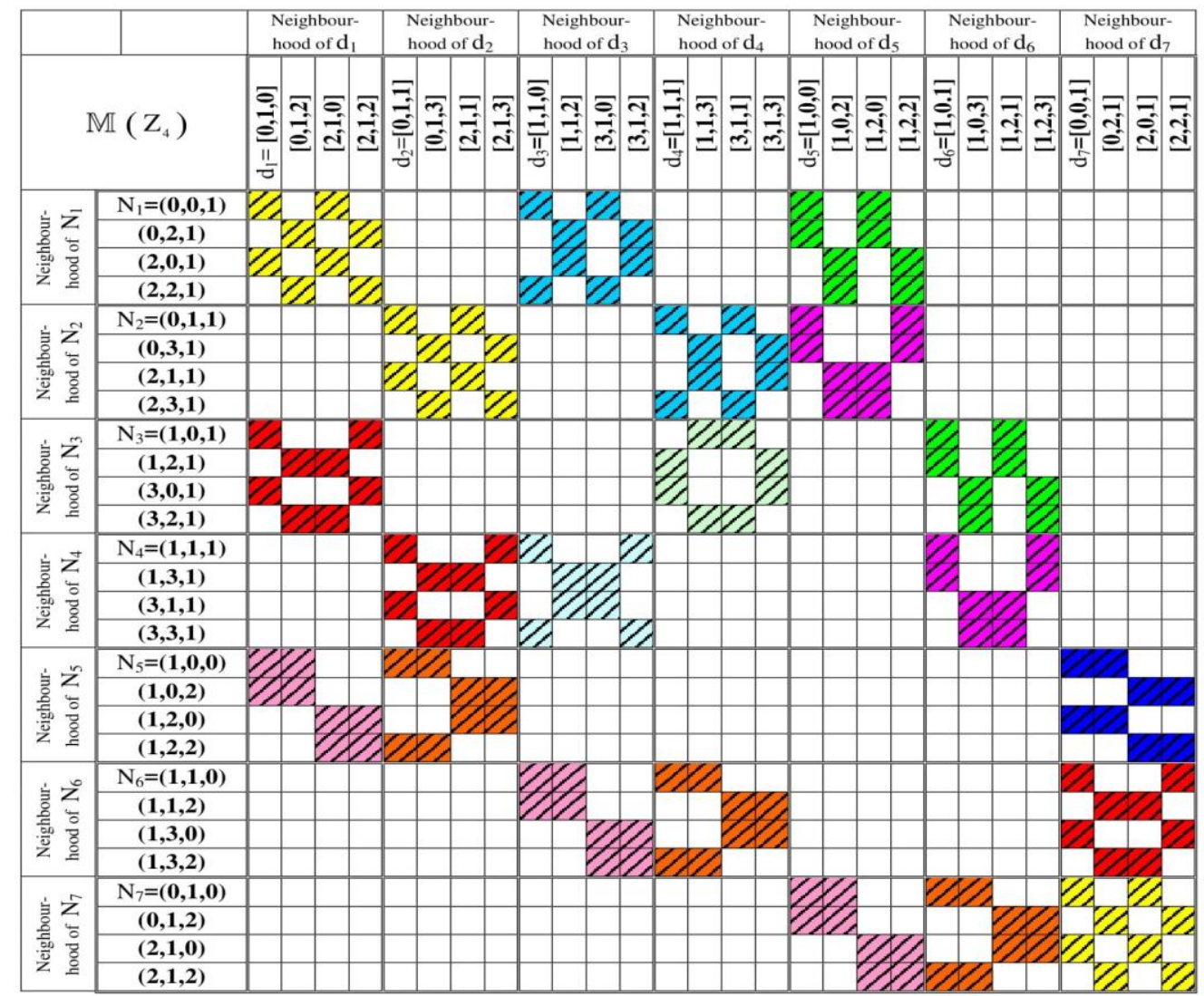

Table 4. The incidence matrix for the plane $\mathrm{M}\left(\mathbb{Z}_{4}\right)$

The number of the collineations of the planes $M\left(\mathbb{Z}_{2}+\mathbb{Z}_{2} \eta\right)$ and $M\left(\mathbb{Z}_{4}\right)$ are $4(7 \cdot 6 \cdot 4)=4(168)=672$ as the number of points in neighborhood of a point is 4 and a basis of the plane consists of the 3-gons. For an arbitrary $\mathrm{m}$, we can generalize this result to $\mathbb{Z}_{p}$ as follows:

The number of the collineations of $\mathrm{M}(\mathbf{A})$ is $\left(p^{m-1}\right)^{2}[v(v-1)(v-(p+1))]$ where $v:=p^{2}+p+1$ is total number of points of a projective plane of order $p$.

Now, we will give an example of a collineation of PK-plane $\mathrm{M}(\mathbf{A})$. This collineation is analogous to the collineation Gs given in [9]. To show that Gs is a collineation, some similar calculations to the proof of [9, Lemma 3] must be done.

For any $s \in \mathbf{A}$, the collineation Gs transforms points and lines as follows:

$$
\begin{aligned}
& (x, y, 1) \rightarrow(x, y+x s, 1), \\
& (1, y, z) \rightarrow(1, y+s, z), \\
& (w, 1, z) \rightarrow\left((1+w s)^{-1} w, 1, z-\left((1+w s)^{-1} w\right)(s z)\right) \\
& {[m, 1, k] \rightarrow[m+s, 1, k]} \\
& {[1, n, t] \rightarrow\left[1,(1+n s)^{-1} n, t-(t s)\left((1+n s)^{-1} n\right)\right],} \\
& {[q, n, 1] \rightarrow[q-s n, n, 1] .}
\end{aligned}
$$

Note that it can be found many collineations, which are similar to this collineation, of the PK-plane. 


\section{Further Results}

It is possible to further generalize all results in this study by taking the local ring $\left(\mathbb{Z}_{\mathrm{p}^{r}}, \mathrm{I}\right)$ instead of the field $\mathrm{F}$ where $\mathrm{p}$ is a prime and $\mathrm{r}$ is a positive integer. In this case, it is clear that $|\mathcal{A}|=\left(\mathrm{p}^{\mathrm{r}}\right)^{\mathrm{m}}$ ve $|\mathbf{I}|=|\mathrm{I}| \times\left(\mathrm{p}^{\mathrm{r}}\right)^{\mathrm{m}-1}=\mathrm{p}^{\mathrm{rm}-1}$ where $\mathcal{A}:=\mathbb{Z}_{p^{r}}+\mathbb{Z}_{p^{r}} \eta+\mathbb{Z}_{p} r \eta^{2}+\cdots+\mathbb{Z}_{p} r \eta^{m-1}$ since $\left|\mathbb{Z}_{p^{r}}\right|=p^{r}$ ve $|I|=p^{r-1}$. So, we can immediately give the following results on the plane $\mathrm{M}(\mathcal{A})$ :

1) The number of points (or lines) in the neighborhood of any point (or any line) is $|\mathbf{I}|^{2}=\left(p^{r m-1}\right)^{2}$, respectively.

2) The number of points of type of $(x, y, 1)$ is $\left(p^{r}\right)^{m} \times\left(p^{r}\right)^{m}=\left[\left(p^{r}\right)^{m}\right]^{2}$,

The number of points of type of $(1, y, z)$ is $\left(p^{r}\right)^{m} \times|\mathbf{I}|$,

The number of points of type of $(w, 1, z)$ is $|\mathbf{I}| \times|\mathbf{I}|=|\mathbf{I}|^{2}$.

Moreover, the results is dually valid for the lines.

3) From 1) and 2) the number of non-neighbour points is:

The number of points of type of $(x, y, 1)$ is $\frac{\left[\left(p^{r}\right)^{m}\right]^{2}}{|r|^{2}}=\frac{\left[\left(p^{r}\right)^{m}\right]^{2}}{\left(p^{r m-1}\right)^{2}}=p^{2}$,

The number of points of type of $(1, y, z)$ is $\frac{\left(p^{r}\right)^{m} \times|I|}{\left|I^{2}\right|^{2}}=\frac{\left(p^{r}\right)^{m}}{|\mathbf{I}|}=\frac{\left(p^{r}\right)^{m}}{p^{r m-1}}=p$,

The number of points of type of $(w, 1, z)$ is $\frac{\left.|I|\right|^{2}}{|r|^{2}}=1$.

Moreover, the results is dually valid for the lines.

4) From 3) the number of non-neighbour points or lines of $\mathrm{PK}$-plane $\mathrm{M}(\boldsymbol{A})$ is $\mathrm{p}^{2}+\mathrm{p}+1$.

5) The total number of the points or the lines of PK-plane $M(\mathcal{A})$ is $|\mathbf{I}|^{2} \times\left(p^{2}+p+1\right)=\left(p^{r m-1}\right)^{2}\left(p^{2}+p+1\right)$.

6) As a result of 4), $M{ }^{\star}$ stated in (PK3) of Definition 4 is a projective plane of order $p$.

7) The number of collineations of PK-plane $M(\mathcal{A})$ is $\left(p^{r m-1}\right)^{2}[v(v-1)(v-(p+1))]$ where $v:=p^{2}+p+1$ is total number of points of a projective plane of order $\mathrm{p}$.

8) Gs is a collineation of PK-plane $\mathrm{M}(\boldsymbol{A})$. Note that it is established similar collineations for the planes.

9) By choosing $p, r$ and $m$, incidence matrix of any line can be obtained by following the previous examples.

\section{Conflicts of Interest}

The authors declare that there are no conflicts of interest regarding the publication of this article.

\section{References}

1. Jukl, M. 1993. "Linear forms on free modules over certain local rings." Acta Univ. Palack. Olomuc. Fac. Rerum Natur. Math. 32: 49-62. 
2. Jukl, M. 1995. "Grassmann formula for certain type of modules." Acta Univ. Palack. Olomuc. Fac. Rerum Natur. Math. 34: 69-74.

3. Erdogan, F.O., Ciftci, S. and Akpınar, A. 2016. "On Modules over Local Rings." Analele Univ. "Ovidius" din Constanta, Math Series, 24(1): 217-230.

4. Ciftci, S. and Erdogan, F.O. 2017. "On projective coordinate spaces." Filomat. 31(4): 941-952.

5. McDonald, B.R. 1976. Geometric algebra over local rings. New York: Marcel Dekker.

6. Hungerford, T.W. 1974. Algebra. New York: Holt, Rinehart and Winston.

7. Nomizu, K. 1966. Fundamentals of Linear Algebra. New York: McGraw-Hill.

8. Baker, C.A., Lane, N.D. and Lorimer, J.W. 1991. "A coordinatization for Moufang-Klingenberg Planes." Simon Stevin, 65: 3-22.

9. Celik, B., Akpinar, A. and Ciftci, S. 2007. "4-Transitivity and 6-Figures in some Moufang-Klingenberg Planes." Monatshefte für Mathematik. 152: 283-294.

10. Akpinar, A. 2010. "The Incidence Matrices For Some Finite Klingenberg Planes." Journal of Balıkesir University Institute of Science and Technology. 12(1): 91-99.

11. Keppens, D. and Van Maldeghem, H. 2009. "Embeddings of projective Klingenberg planes in the projective space PG(5,K)." Beiträge zur Algebra und Geometrie. 50(2): 483-493.

12. Keppens, D. 2017. 50 years of Finite Geometry, the "Geometries over finite rings" part, Innovations in Incidence Geometry. 15: 123-143.

13. Kleinfeld, E. 1959. "Finite Hjelmslev planes." Illinois J. Math. 3(3): 403-407.

14. Drake, D.A. and Lenz, H. 1975. "Finite Klingenberg Planes." Abh. Math. Sem. Hamburg. 44: 70-83.

15. Drake, D.A. and Jungnickel, D. 1985. "Finite Hjelmslev Planes and Klingenberg Epimorphisms." In: R. Kaya, P. Plaumann and K. Strambach (eds), Rings and Geometry. NATO ASI Series (Series C: Mathematical and Physical Sciences), vol 160. Dordrecht: Springer. 